\title{
Expert Knowledge Correlated Evaluation of Intrusion Detection System in Heterogeneous IoT
}

\author{
Nitish A, J. Hanumanthappa, S.P. Shiva Prakash*, and Kirill Krinkin
}

\begin{abstract}
The dynamic heterogeneous IoT contexts adversely affect the performance of learning-based network intrusion detection and prevention systems resulting in increased misclassification rates-necessitating an expert knowledge correlated evaluation framework. The proposed framework includes intrusion root cause analysis and a correlation model that can be generalized over any network intrusion dataset, corresponding expert knowledge, detection technique, and learning-based algorithm. The experimentations prove the robustness of the propounded framework on imbalanced datasets.
\end{abstract}

Index Terms-Context awareness, correlated evaluation metrics, expert knowledge correlation, heterogeneous IoT, learningbased network intrusion detection and prevention, root cause analysis

\section{INTRODUCTION}

$\mathbf{H}$ ETEROGENEOUS networks that offer ad hoc networklike flexibility and IoT network-like connectivity are suitable for ubiquitous computing and proliferating content consumption in critical applications across domestic and industrial space [1]-[3]. Such networks are called heterogeneous IoT (or HetIoT) [5].

Learning-based network intrusion detection systems (NIDS) for such HetIoT infrastructures suffer from high misclassification rates [1], [8] due to context-unaware statistical evaluation of (machine or deep) learning-based algorithms as HetIoT involves dynamic contexts. Such dynamic scenarios necessitate reinforcing an expert knowledge (EK) framework for context-aware decision-making and minimizing false alerts [2], [4], [6], [7] to avoid high recovery costs due to failure. EK can include information like high-level traffic behaviors, constituting device specifications, and operating conditions.

We propose a generalized EK correlated evaluation framework with correlated metrics that performs well on imbalanced data and can supplement any (machine or deep) learningbased algorithm to obtain reduced false alerts for any intrusion detection (ID) application, provided a relevant expert knowledgebase for the network traffic dataset is available. Furthermore, this framework permits policy distribution across

This work is supported by the Ministry of Science and Higher Education of the Russian Federation under the Grant number 075-15-2020-933. (Corresponding author: S.P. Shiva Prakash)

Nitish A (e-mail: nitish.anantha@acm.org) and J. Hanumanthappa (e-mail: hanumsbe@gmail.com) are with the Department of Studies in Computer Science, University of Mysore, India.

* S.P. Shiva Prakash (e-mail: shivaspasjce.ac.in) is with the Department of Information Science \& Engineering, JSS Science and Technology University, Mysuru, India.

Kirill Krinkin (e-mail: kirill@krinkin.com) is with the Department of Software Engineering and Computer Applications, St. Petersburg Electrotechnical University "LETI," Russia. the HetIoT subnets for context-specific near real-time intrusion detection and prevention.

We evaluate our proposition on the SVM algorithm by employing the Bot-IoT dataset and constructing a relevant expert knowledge base with the following contributions:

1) A high-level EK framework (RCA table) for intrusion root cause analysis.

2) A reinforcement method (Correlation algorithm) for correlating ID alerts with EK inferences for reducing false alerts.

3) Correlated evaluation metrics derived from standard ID evaluation metrics based on the Correlation algorithm.

\section{RELATED WORK}

The existing NIDPS policies for heterogeneous networks [1], [3], [4] suffer from high misclassification rates due to context-unaware traffic processing by learning-based algorithms, adversely affecting the HetIoT performance.

Researchers have proposed means to reduce misclassifications involving threat context computation [4] and intrusion root cause analysis [8]. However, such works employ ontological techniques [7] that are computation-heavy [1] and provide no information on the decentralization of policies, affecting the real-time performance of HetIoT-NIDPS especially during big data processing.

\section{Correlated Evaluation Framework}

The dynamic contexts in a HetIoT adversely affect the context unaware low-level statistical inferences of ID algorithms. Thus, reinforcing them with the extracted high-level behavioral descriptions improves network redesign that is context-aware and resistant to frequent security compromises, benefitting from the interpretability of knowledge-driven specification and the adaptability of data-driven detection [1]. To achieve this, we propose an EK framework.

\section{A. RCA Expert Knowledge Framework}

The proposed RCA framework offers a high-level intuition for identifying faults in the HetIoT behavior for a threat context with potential attacks and analyzes root causes of the intrusions encountered. Such a setting is helpful for easier network maintenance, as it reduces examining the network logs. Moreover, interpreting network logs from heterogeneous devices is never straightforward and requires additional computations and time to normalize.

The framework contains the RCA table (Table I), offering a list of possible faults for an attack category compiled by 
domain experts, where an attack is associated with a high-level unexpected network behavior. In addition, the NID dataset [3] offers information about such behaviors-identified as specific faults and their probable causes that include system vulnerabilities. We use this information obtained from multiple repositories as the EK to correlate ID alerts.

\section{B. Misuse Detection Model}

Misuse detection classifies the network traffic instances according to the class labels.

Definition III.1 (Misuse Detection). Let $D=\left\{d_{t}, y_{t}\right\}_{t=1}^{M}$ be the data-driven information (dataset). The prediction (binary classification) that an instance belongs to an attack class $C_{i} \in$ $C$ where $C=\{0,1\}$ is,

$$
\mathrm{P}\left(f\left(d_{t}\right)=1 \mid C_{i}\right)
$$

We employ the proposed Correlation model (Algorithm 1) to evaluate the $S V M$ classifier alerts and obtain correlated alertsresulting in SVM-Corr classifier. The Correlation algorithm is generic and is independent of learning-based algorithms, NID datasets and corresponding EK, and ID techniques.

\section{Correlation Model}

The Correlation model correlates the ID alerts with the EK inferences from the RCA table.

Definition III.2 (Correlation). Let $E_{R C A}(k) \forall k \in K$ be an expert knowledge instance of a dataset instance $d \in D$. Let $\mathcal{E}:(d, k) \rightarrow C$ be an evaluation function such that, evaluating the dataset and the corresponding expert knowledge instances helps detect an attack label instance. i.e.,

$$
\mathcal{E}\left(d_{q}, k_{r}\right)=C_{i}
$$

Therefore, the ID alerts and the corresponding EK inferences are correlated (i.e., yield same class labels) iff,

$$
\mathrm{P}\left(f\left(d_{q}\right)\right) \longleftrightarrow E_{R C A}\left(k_{r}\right)
$$

1) Correlation Algorithm: Algorithm 1 offers the pseudocode for correlation-analogous to equation (3). Since $E_{R C A}$ informs only about the attacks present in the system, it always checks for $T P$ and $F N$. Thus, uncorrelated ID alerts lead to $F P$-analyzed and correctly classified as correlated $T N(C T N)$ and $F N$-analyzed and correctly classified as correlated TP $(C T P)$ through Algorithm 2, which is analogous to equation (2) and results in reduced false alerts.

The correlated alerts with labels and the EK inferences are updated to the knowledgebase (Fig. 1). Finally, the correlation algorithm outputs the alerts described in Table II.

2) Correlated Intrusion Detection Metrics: We formulate the correlated ID metrics from the results of Correlation algorithm. We derive the proposed metrics from the standard classification metrics of a learning-based algorithm.

The correlation error $\xi$ due to inefficiency in preparing the RCA table $E_{R C A}$ (i.e., unavailability of corresponding EK instance for an ID attack instance) results in missed
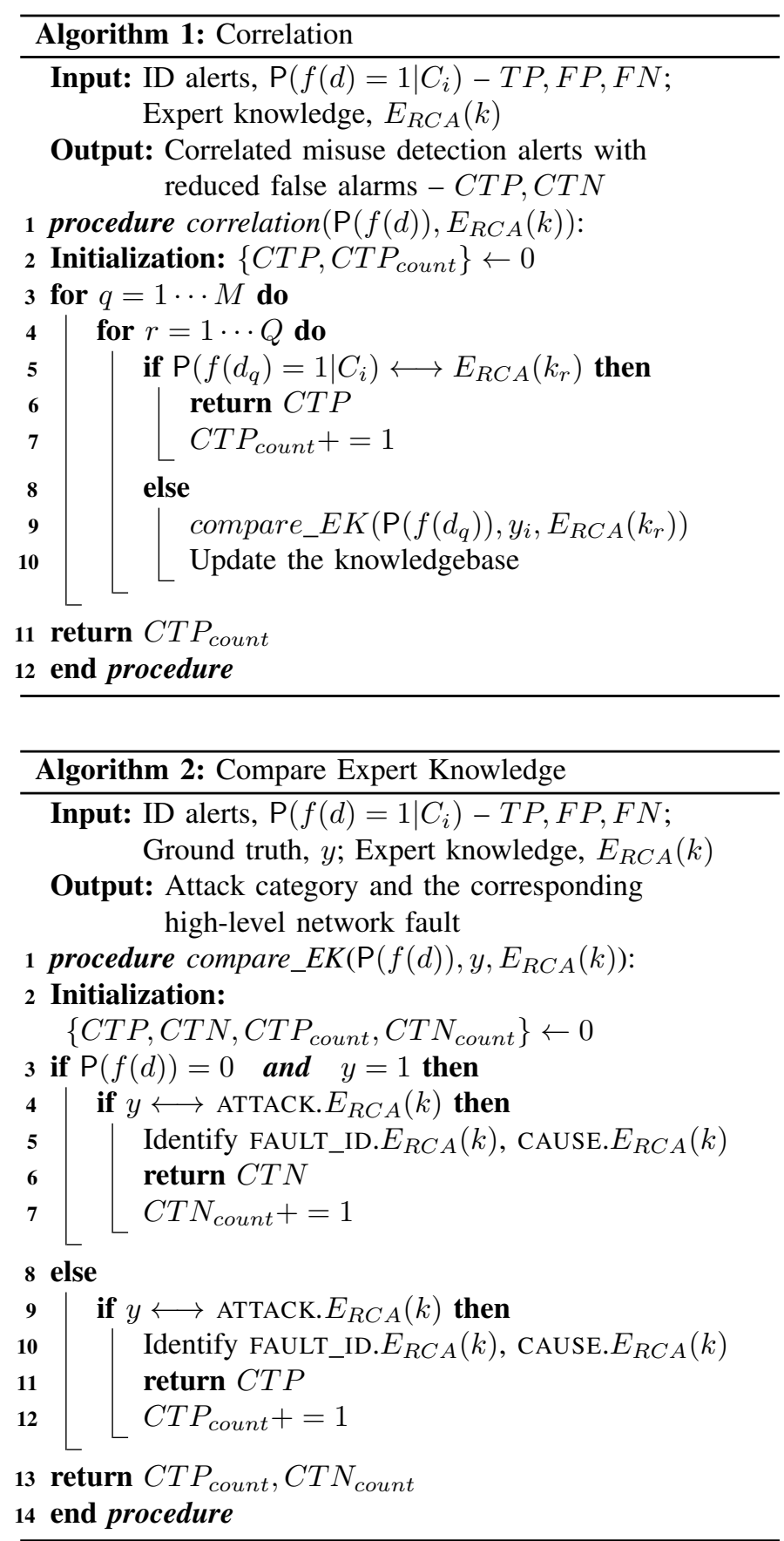

false ID alerts $(F N$ and $F P)$-reducing the metric scores. Such misclassifications upon further examination lead to new HetIoT behaviors updated in the expert knowledgebase.

$$
\xi=\xi_{F P}+\xi_{F N}
$$

Therefore, we formulate the correlated ID evaluation metrics as follows:

- Correlated Accuracy $(\mathcal{A})$ :

$$
\begin{aligned}
\text { Accuracy } & =\frac{T P+T N}{T P+T N+F P+F N} \\
\Rightarrow \mathcal{A} & =\frac{C T P+T N}{C T P+T N+C T N+\xi}
\end{aligned}
$$


TABLE I

A SAMPle of AtTack Behaviors-wise Root Cause Analysis (RCA) Framework

(REFER TO THE CONCLUSION SECTION FOR THE DETAILED VERSION)

\begin{tabular}{|l|l|l|l|l|}
\hline Attack & Behavior & Fault & $\begin{array}{l}\text { Fault } \\
\text { ID }\end{array}$ & Cause \\
\hline \hline DoS & $\begin{array}{l}\text { Large incoming network traffic } \\
\text { from a HetIoT client to a server } \\
\text { IP, Disrupted services }\end{array}$ & $\begin{array}{l}\text { Apache web server receiving client requests with } \\
\text { many HTTP headers (around 10,000), Server } \\
\text { load average } \geq 5 \text { or more, Out of memory } \\
\text { HTTPD daemon (apache2) }\end{array}$ & $\begin{array}{l}\text { Legitimate HTTP request headers } \\
\leq 20\end{array}$ \\
\hline Recon & $\begin{array}{l}\text { Large incoming network traffic } \\
\text { across multiple HetIoT clients } \\
\text { from single or multiple external } \\
\text { IPs within a specific time window }\end{array}$ & $\ldots$ & R1 & $\begin{array}{l}\text { Device replying before a threshold } \\
\text { of requests exposes its IP }\end{array}$ \\
\hline$\ldots$ & $\ldots$ & $\ldots$ & $\ldots$ & $\ldots$ \\
\hline
\end{tabular}

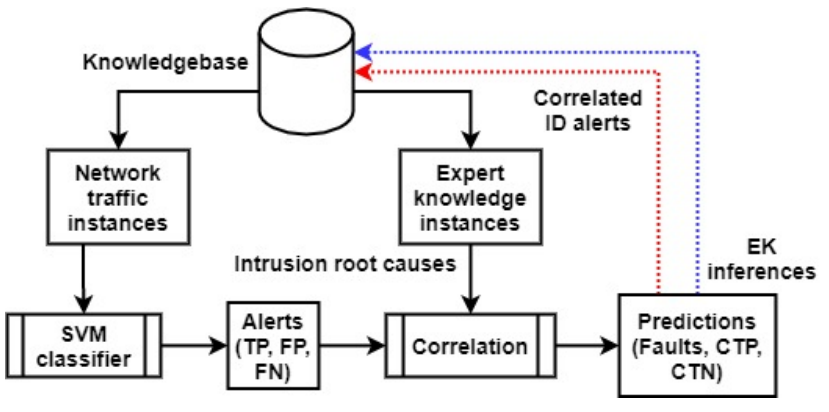

Fig. 1. Proposed correlated NIDS architecture

TABLE II

STANDARD ID ALERTS AND THEIR CORRESPONDING CORRELATIONS

\begin{tabular}{|c|c|}
\hline Classification Alerts & Correlated Alerts \\
\hline \hline True Positive $(T P)$ & Correlated True Positive $(C T P)$ \\
\hline True Negative $(T N)$ & - \\
\hline False Positive $(F P)$ & Correlated True Negative $(C T N)$ \\
\hline False Negative $(F N)$ & Correlated True Positive $(C T P)$ \\
\hline
\end{tabular}

- Correlated Precision $(\mathcal{P})$ :

$$
\begin{aligned}
\text { Precision } & =\frac{T P}{T P+F P} \\
\Rightarrow \mathcal{P} & =\frac{C T P}{C T P+C T N+\xi_{F P}}
\end{aligned}
$$

- Correlated Recall $(\mathcal{R})$ :

$$
\begin{aligned}
\text { Recall } & =\frac{T P}{T P+F N} \\
\Rightarrow \mathcal{R} & =\frac{C T P}{C T P+\xi_{F N}}
\end{aligned}
$$

- Correlated F1-score $(\mathcal{F})$ :

$$
\begin{aligned}
F 1-\text { score } & =2 \times \frac{\text { Precision } \times \text { Recall }}{\text { Precision }+ \text { Recall }} \\
\Rightarrow \mathcal{F} & =2 \times \frac{\mathcal{P} \times \mathcal{R}}{\mathcal{P}+\mathcal{R}}
\end{aligned}
$$

\section{Algorithm Analysis}

We perform formal analyse ${ }^{1}$ of the Correlation algorithm to explore its properties and prove that it can offer near real-time performance.

\footnotetext{
${ }^{1}$ Refer to the Conclusion section for proofs.
}

Theorem IV.1 (Time Complexity of Correlation Algorithm). The worst-case time complexity of the Correlation algorithm is $\mathcal{T}_{\text {correlation }} \rightarrow \mathcal{O}(M Q)$.

Corollary IV.1.1 (Average Correlations). The average correlations of the Correlation algorithm linearly reduce to $\mathcal{T}_{\text {correlation }} \rightarrow \mathcal{O}(M)$.

Definition IV.1 (Generalizability of Correlation Model). Since the Correlation model utilizes context-specific expert knowledge from a network traffic data of any attack type, it can supplement any (machine or deep) learning-based algorithm for supervised misuse detection or unsupervised anomaly detection, provided a suitable expert knowledgebase is available.

Hence, the proposed algorithm can adapt to any intrusion detection technique and detection algorithm, independent of network traffic and expert knowledge data.

Thus, we say that the Correlation model is generalized over network traffic dataset $\mathrm{D}$, expert knowledge $\mathrm{E}$, intrusion detection technique ID, and detection algorithm A. Therefore,

$$
\text { Correlation : } \mathcal{G}(\mathrm{D}, \mathrm{E}, \mathrm{ID}, \mathrm{A})
$$

\section{EXPERIMENTAL ANALYSIS AND DISCUSSION}

We begin by preparing the dataset for classification, then perform misuse detection using the SVM algorithm. We then correlate the misclassified alerts from SVM through the Correlation algorithm (SVM-Corr classifier) to obtain the correlated results with minimized false alerts.

Finally, we perform binary classification with SVM-Corr by splitting the dataset into Normal and four attack classes (DoS, DDoS, Recon, and Theft). We choose this setting to distribute the attack-wise trained classifier models across the HetIoT according to each subnet's specific threat (attack) contexts.

The experimental setup is according to the specifications in the scikit-learn ${ }^{2}$ machine learning library.

\section{A. Experimental Environment}

We list the experimental settings-including the hardware specifications and the algorithmic parameters used for the work in Table III.

\footnotetext{
${ }^{2}$ https://scikit-learn.org/stable/
} 
TABLE III

EXPERIMENTAL ENVIRONMENT SETTINGS For Misuse Detection

\begin{tabular}{|l|l|}
\hline Parameter & Specification \\
\hline \hline CPU & AMD with 6 cores $(3.0 \mathrm{GHz})$ \\
\hline RAM & 24 GB (3200 MHz) \\
\hline Detection algorithms & SVM, SVM-Corr \\
\hline SVM settings & $\begin{array}{l}\text { LinearSVC, CV }=4, \\
\text { max_iter }=100,000\end{array}$ \\
\hline
\end{tabular}

\section{B. Dataset Preprocessing}

We preprocess the 10-best features CSV file of the Bot-IoT dataset using the Label and one-hot encoding techniques to convert all the categorical features (like protocol, ip address, port, and class label) into numericals for two types of binary classification:

- Normal vs. Attack

- Normal vs. \{DoS, DDoS, Reconnaissance, Theft $\}$

1) Dataset Normalization: We scale the encoded dataset features for classification. First, the technique standardizes the feature instances by removing the mean and scaling them to unit variance, as required by the SVM algorithm.

\section{Results and Analysis}

We present the details of the experiments conducted and the results obtained, supporting our claims in previous sections.

1) SVM-Corr Classifier: The SVM-Corr classifier makes better predictions with lower false alarm rates, improving classification performance.

We experiment to evaluate the proposed approach's performance compared to the existing work [3] by setting up the Linear SVM classifier with 4-fold cross-validation and max iterations set to 100,000 (for convergence). However, in both cases, the classifier suffered from longer training times (in the order of $10^{3}$ seconds). Table IV presents the results.

TABLE IV

SVM AND SVM-CORR ClassiFIER PERFORMANCES (IN \%) ON THE COMPLETE Dataset (NoRMAL vs. ATtACK With $\mathrm{CV}=4$ )

\begin{tabular}{c|c|c}
\hline Classifier & Metric & Result (\%) \\
\hline \hline SVM-Corr & $\mathcal{A}$ & $\mathbf{9 9 . 9 9 5 1}$ \\
SVM & Accuracy & 88.3727 \\
\hline SVM-Corr & $\mathcal{P}$ & $\mathbf{9 9 . 9 9 7 4}$ \\
SVM & Precision & $\mathbf{1 0 0}$ \\
\hline SVM-Corr & $\mathcal{R}$ & $\mathbf{9 9 . 9 7 4 0}$ \\
SVM & Recall & 88.3712 \\
\hline
\end{tabular}

We split the Bot-IoT dataset based on the existing attack types and present the corresponding class-wise (Normal and 4 Attacks) distribution in Table V.

Table VI presents the comparative performances of the SVM and SVM-Corr classifiers on the class-wise distributed imbalanced dataset—indicating improved performance.

\section{CONCLUSION}

We present an RCA EK correlated NIDS evaluation framework generalizable across multiple NID techniques, algorithms, datasets, and corresponding EK. Furthermore, this
TABLE V

Class-wise Bot-IoT TrafFic SAMPLES Distribution

\begin{tabular}{|c|c|c|}
\hline Class & Train set & Test set \\
\hline \hline Normal & 370 & 107 \\
DoS & 1320148 & 330112 \\
DDoS & 1541315 & 385309 \\
Reconnaissance & 72919 & 18163 \\
Theft & 65 & 14 \\
\hline
\end{tabular}

TABLE VI

ATtaCK-Wise SVM ANd SVM-CORR Classifier PERFormances (IN $\%)$ ON THE NORMALLY DISTRIBUTED IMBALANCED BOT-IOT DATASET

\begin{tabular}{c|c|c|c|c|c}
\hline Classifier & Attack & Accuracy & Precision & Recall & F1-score \\
\hline \hline \multirow{3}{*}{ SVM } & DoS & 99.9821 & 99.9879 & 99.9942 & 99.9911 \\
& DDoS & 97.8755 & 99.9920 & 97.8829 & 98.9262 \\
& Recon & 98.4925 & 99.9832 & 98.5089 & 99.2405 \\
& Theft & 19.7917 & $\mathbf{1 0 0}$ & 19.7917 & 33.0435 \\
\hline & & $\mathcal{A}$ & $\mathcal{P}$ & $\mathcal{R}$ & $\mathcal{F}$ \\
\hline \hline \multirow{3}{*}{ SVM-Corr } & DoS & $\mathbf{9 9 . 9 9 8 5}$ & $\mathbf{9 9 . 9 9 9 4}$ & $\mathbf{9 9 . 9 3 7 6}$ & $\mathbf{9 9 . 9 9 9 7}$ \\
& DDoS & $\mathbf{9 9 . 9 9 7 9}$ & $\mathbf{9 9 . 9 9 9 7}$ & $\mathbf{9 9 . 8 1 0 4}$ & $\mathbf{9 9 . 9 9 9 8}$ \\
& Recon & $\mathbf{9 9 . 9 8 3 4}$ & $\mathbf{9 9 . 9 9 4 5}$ & $\mathbf{1 0 0}$ & $\mathbf{9 9 . 9 9 7 2}$ \\
& Theft & $\mathbf{1 0 0}$ & $\mathbf{1 0 0}$ & $\mathbf{1 0 0}$ & $\mathbf{1 0 0}$ \\
\hline
\end{tabular}

framework results in near real-time performance and improved efficiency on imbalanced datasets.

In our future work, we attempt to qualify the impact of attack alerts in a HetIoT threat context by defining a Fault Score metric for the RCA table for alert prioritization and threat (attack) severity assessment.

Link for the supplementary information (with the detailed RCA table and algorithm analyses):

https://drive.google.com/drive/folders/ 1gZ5WObZlApszoxq83iFHnY71GqgBMVKL? usp= sharing

\section{REFERENCES}

[1] B. Steenwinckel et al, "FLAGS: A methodology for adaptive anomaly detection and root cause analysis on sensor data streams by fusing expert knowledge with machine learning," Future Generation Computer Systems, vol. 116, pp. 30-48, 2021.

[2] D.M.A. Da Silva and R.C. Sofia, "A Discussion on Context-Awareness to Better Support the IoT Cloud/Edge Continuum," IEEE Access, vol. 8, pp. 193686-193694, 2020.

[3] N. Koroniotis et al, "Towards the development of realistic botnet dataset in the Internet of Things for network forensic analytics: Bot-IoT dataset," Future Generation Computer Systems, vol. 100, pp. 779-796, 2019.

[4] M. Trnka et al, "Securing Internet of Things Devices Using the Network Context," IEEE Transactions on Industrial Informatics, vol. 16(6), pp. 4017-4027, 2019.

[5] T. Qiu et al, "How Can Heterogeneous Internet of Things Build Our Future: A Survey," IEEE Communications Surveys \& Tutorials, vol. 20 , no. 3, pp. 2011-2027, 2018.

[6] O.B. Sezer, E. Dogdu, and M. Ozbayoglu, "Context Aware Computing, Learning and Big Data in Internet of Things: A Survey." IEEE Internet of Things Journal, vol. 5(1), pp. 1-27, 2018.

[7] G. Xu et al, "Network security situation awareness based on semantic ontology and user-defined rules for Internet of Things," IEEE Access, vol. 5, pp. 21046-21056, 2017.

[8] S.O. Al-Mamory and H. Zhang, "Intrusion detection alarms reduction using root cause analysis and clustering," Computer Communications, vol. 32, pp. 419-430, 2009. 